\title{
Entre présentation et représentation : le corps à travers l'œil de la caméra de Carolee Schneemann
}

\author{
Marie-Laure Delaporte \\ Université Paris Ouest Nanterre La Défense, France
}

Dès le début des années 1960, la peintre américaine Carolee Schneemann s'empare de la caméra pour filmer ses performances. Ainsi l'artiste, comme pour s'émanciper des collègues masculins avec lesquels elle travaille - Stan Brakhage, Robert Morris ou Anthony McCall, entre autres -, ajoute l'outil filmique à ses autres pratiques artistiques que sont la peinture ou la performance. Faisant partie des fondatrices et fondateurs du groupe polymorphe du Judson Dance Theater, fréquentant les artistes Fluxus, revendiquant des préoccupations politico-sociales telles que le féminisme ou l'anti-militarisme, Schneemann utilise la caméra à la fois dans une volonté de décloisonner les genres artistiques, d'affirmer une identité créatrice et féminine et de repousser les limites de la représentation de son propre corps à travers une affirmation de son image et de sa sexualité. En 1965, Robert Morris met en scène la performance Site dans laquelle Schneemann incarne le symbole féminin dans cette interprétation performative du tableau moderniste Olympia d'Edouard Manet (1863). Il est intéressant de noter que Morris choisit une œuvre considérée comme l'un des premiers tableaux modernes pour définir sa propre modernité artistique à travers le genre de la performance. Pourtant Schneemann se trouve reléguée au second plan, passive, allongée, nue, sur un sofa, alors que Morris est celui qui effectue l'action. Catherine Elwes constate que « dans le contexte de la performance, l'objectif majeur était de refuser la double insulte d'être relégué soit à la catégorie de l'actrice/prostituée de théâtre, attisant les désirs sexuels du public, ou de la muse/modèle, figée dans une pose lascive apportant inspiration à l'artiste masculin ». Incarnant ces deux symboles, Schneemann décide alors d'orienter sa propre pratique artistique dans une dynamique du décadrage, du décentrement et dépassant la performance sculpturale de Morris. En 1992, dans une lettre à Dorothea Rockbrune, elle avoue d'ailleurs son sentiment au sujet de Site, si les rôles avaient été inversés:

Bob aurait été sur ce petit banc avec un col en velours autour du cou pendant que moi j'aurais soulevé et déplacé ces rectangles de contreplaqué. L'expérience d'être immobilisée et historicisée par Bob était l'une des multiples approches de ma 'collaboration' avec mes amis artistes masculins, au cours desquelles mes propres appréhensions et énergies étaient étouffées, ou détournées vers des représentations invraisemblables pendant les années 1960 (Stiles Correspondance 413) ${ }^{1}$

Schneemann fait donc le choix de s'emparer de la présentation et de la représentation de son propre corps à travers les médiums avec lesquels elle développe ses recherches et de se saisir de son pouvoir de création. Il est tout à fait probable que cette volonté et cette capacité à expérimenter avec les

\footnotetext{
${ }^{1}$ « Bob would be on the little shelf with a velvet collar around his neck while $I$ lifted, moved the heavy 6' $\mathrm{x} 8$ ' $\mathrm{x} 2$ " 'plywood rectangles. The experiment in being immobilized and historicized by Bob, was one of several approaches to "collaboration" with men artist friends, in which my own meanings, energies were held captive, or diverted to some unlikely representation during the 1960s ». En l'absence de traduction officielle, nous traduisons toutes les citations originales présentes dans cet article.
} 
techniques, et notamment filmiques, viennent de la frustration ressentie lors de ces premières collaborations. Contrairement à ces performances, dans lesquelles la femme reste une image/objet, Emma Lavigne note que Schneemann a pour objectif de construire un discours autour du corps différent de ses prédécesseurs : « elle cherche à mettre en avant la force politique du corps, qui devient le lieu du discours, la scène privilégiée de la remise en question des tabous et des stéréotypes liés à la représentation du corps des femmes et à l'ordre patriarcal » (Lavigne 236).

Influencée par les théories de Wilhelm Reich, ${ }^{2}$ l'ouvrage Le Théâtre et son double d'Antonin Artaud ${ }^{3}$ ou encore les ouvrages de Simone de Beauvoir, ${ }^{4}$ Schneemann explique que les « femmes artistes explorent l'imagerie érotique car leur corps incarne un champ de bataille historique » (Schneemann Seasonal 217). ${ }^{5}$ L'artiste fait siens ces différents préceptes en développant le principe «Eye/Body » : un corps à la fois instrument de vision, créateur et objet de la création, permettant « aux spectateurs de confronter leur identité et attitude à leur propre genre et celui des autres » (Schneemann Imaging 45). ${ }^{6}$ Dans cette démarche, la caméra devient un outil primordial: «Le processus d'enregistrement, écrit Mélissa Ragona, n'était pas une simple action mimétique de copie, mais un événement qui fut - immédiatement - structuré et imprévisible. Pour Schneemann, non seulement l'œil, mais aussi le corps doivent enregistrer afin que l'événement se produise » (Ragona 35). ${ }^{7}$ Ainsi, le corps est utilisé tant dans une démarche politique que dans une réflexion protoféministe et poétique remettant en question les interdits culturels collectifs. L'artiste associe film, performance et références autobiographiques en exprimant une intimité physique comme engagement dans une confrontation au genre qui passe notamment par la mise en scène de la nudité corporel de l'artiste. Dépassant les interdits sociaux, elle privilégie un aspect transgressif de la nudité féminine en opposition à la tradition artistique du nu féminin. Dan Cameron explique que «Schneemann et les artistes féminines de sa génération étaient marginalisées en conséquence de leur usurpation des privilèges masculins au sein des règles de la représentation $\gg$ (Cameron 12) ${ }^{8}$ La performance et le film permettent aux artistes de se ré-approprier leur corps et leur identité.

Lors d'un entretien, l'artiste affirme qu'en 1963, elle envisageait déjà la possibilité de recréer l'image corporelle, « en utilisant le corps, en soustrayant le corps féminin aux mains des traditions masculines de l'histoire de l'art » (Aquin 160). A travers la performance et le film, Schneemann parvient à s'imposer à la fois comme artiste et femme au cours de la décennie 1960. Les

\footnotetext{
${ }^{2}$ Dans Die Sexualität im Kulturkampf, publié en 1936, le psychanalyste Wilhelm Reich y développe sa théorie des pulsions et répressions sexuelles pouvant mener à la violence individuelle et de masse.

${ }^{3}$ Carolee Schneeman reprend notamment le principe d'immédiateté et le rapport direct au public développé par Antonin Artaud dans Le Théâtre et son double, série d'essais publié en 1938.

${ }^{4}$ Simone de Beauvoir explique le principe de la femme comme «autre » dans Le Deuxième sexe, publié en 1949 et traduit en anglais en 1953, dont Schneemann a connaissance.

5 « Women artists explore erotic imagery because our bodies exemplify a historic battleground. $»$

${ }^{6}$ « viewers to confront identifications and attitudes toward their own and the other's gender. »

${ }^{7}$ « The process of 'recording' was not a simple mimetic task of copying, but rather an event that was - at once - structured and unpredictable. For Schneemann, not only the eye, but also, the body had to record in order for an 'event' to occur. »"

8 « Schneemann and the women artists of her generation were marginalized as a direct result of their successful usurpation of male privilege within the rules of representation. »
} 
femmes, comme le rappelle David James, ont eu un rôle déterminant dans le cinéma indépendant et d'avant-garde comme le prouve notamment l'œuvre de Maya Deren (James 313). Ces artistes n'hésitent pas à s'emparer des transgressions sociales, culturelles, à remettre en question les différences arbitraires de sexe et de genre et à utiliser ainsi le corps et la sexualité comme terrain de contestation.

Comment le film va-t-il donc participer à la reconstruction de l'image d'un corps artistique féminin? Le travail de Schneemann connaît une progression partant du développement du théâtre cinétique pluridisciplinaire, permettant par la suite une concentration sur la spécificité du médium filmique, pratiques réaffirmant les interrogations artistico-féministes ambiguës de l'artiste.

Puisant ses références tant dans les théories de D'Arcy Thompson que dans celles du formaliste Henri Focillon, Schneemann explique que les substances ne sont pas interchangeables, mais les techniques se pénètrent entre elles, et à ce moment même, les interférences tendent à créer de nouvelles substances (Schneemann More Than Meat 7).

\section{La recherche du mouvement à travers le théâtre cinétique}

Cette volonté d'interdisciplinarité se développe dès la fin des années 1950 dans ce que l'artiste nomme "le théâtre cinétique », une recherche du mouvement dans toutes les disciplines artistiques contemporaines, à la fois peinture, photographie, film et performance. D'après Kenneth White: « Le théâtre cinétique était le terme de Schneemann pour son mode de production hétérogène dans Meat Joy et d'autres travaux de cette période. Le théâtre cinétique était un processus d'intervention contre les idées culturelles répressives, en particulier la relégation des femmes à la béatitude domestique » (White 290). ${ }^{9}$ Ce que Schneemann nomme ses œuvres de Kinetic Theater sont ses performances collectives au sein desquelles elle recherche les dynamiques du mouvement. Un assemblage temporaire d'entités humaines, technologiques et matérielles configurées en tant qu'art étant également un événement ... l'artiste s'adressant directement au public » (Elwes 66). ${ }^{10}$ Mais plus encore, c'est le principe d'immédiateté qui retient l'attention de la jeune performeuse à l'époque: un rapport direct au corps, un investissement sans limite face au lieu et au public. Dès lors, le corps est considéré comme une unité potentielle de mouvement. Le film permet l'exploration d'une « image en mouvement », selon un processus de création non-déterminé, imprévisible, involontaire, faisant apparaître d'autres attributs. Le corps est un véritable matériau au service de l'artiste dans la création de son image. Schneemann met en pratique cette théorie dès 1964 avec Meat Joy, ${ }^{11}$ en réponse à l'invitation de Jean-Jacques Lebel à créer une performance pour le Festival de la Libre Expression, à Paris, le 29 mai de cette même année. ${ }^{12}$ Mais dès la première représentation, le problème de la

\footnotetext{
9 « Kinetic theater was Schneemann's term for her heterogeneous mode of production in Meat Joy and other works of that period. Kinethic theater was a process of intervention against suppressive cultural conceits, in particular the relegation of women domestic bliss. »

10 « a temporary assemblage of human, technological and material entities configured as art is also an event ... the direct address of an artist to a live audience. »

${ }^{11}$ Meat Joy est une performance collective, créée en 1964, d'une durée de 60 à 80 minutes. Les performeurs se couvrent de poisson, de poulet, de saucisses, de peinture humide, de plastique, de corde et de papier.

${ }^{12}$ La performance fut également présentée le 8 juin 1964 au Dennison Hall à Londres et les 16, 17 et 18 novembre 1964 au Judson Church de New York
} 
mise en présence directe de la nudité des corps avec le public est flagrant. ${ }^{13} \mathrm{La}$ performance collective intitulée Meat Joy met en scène les interactions humaines au sein des changements physiques, au cœur d'une atmosphère onirique, semi-inconsciente, d'un rite érotico-performatif où la chair se fait matériau. Dans ses notes l'artiste écrit: « La concentration n'est jamais sur soi, mais sur les matériaux, les gestes et les actions qui nous incluent. Un sens que nous devenons ce que nous voyons, ce que nous touchons. Une certaine tendresse (ou empathie) est envahissante - et les actions les plus violentes: couper, émincer, lancer des poulets par exemple $\gg .{ }^{14}$

Chargée d'ambiguïté et d'équivoque, la performance est formée de mouvements chorégraphiés, répétés qui évoluent de façon improvisée au fil de son déroulement, permettant de dépasser les limites à la fois spatiales et symboliques avec le public. Meat Joy émane directement de la formation picturale de l'artiste à travers l'utilisation de la "matière-peinture » dont se couvrent les performeurs apportant une picturalité supplémentaire à l'œuvre qui adopte diverses formes : la matérialité, le cinétisme, la visualisation picturale, temporelle et spatiale. Le corps se voit envisagé comme un matériau tactile intégrant une forme artistique pouvant être qualifiée d'expanded painting. En effet l'artiste qui se revendique avant tout comme peintre: "Je suis un peintre qui étend les principes visuels de la peinture dans le temps et dans l'espace » (Ténèze 109); ${ }^{15}$ à travers le film et la performance, sort des limites du cadre et affirme son intérêt pour la texture, la matérialité et la tactilité picturales, pour le mouvement et le temps dans sa propriété durationnelle. Au contraire de la manipulation du corps féminin qui a pu être faite par des artistes masculins, comme c'est le cas d'Yves Klein dans les Anthropométries, Schneemann tente d'affirmer une liberté créatrice et féminine. A travers cette œuvre de théâtre cinétique, ${ }^{16}$ Schneemann met en valeur les sensations corporelles émanant de la performance collective. C'est à la fois une mise en espace et une mise en scène des corps relevant de la recherche du mouvement qui prend forme dans le film réalisé lors de la première représentation à Paris. ${ }^{17}$ Meat Joy met ainsi en scène huit performeurs, hommes et femmes, à moitié nus, transformés en véritables sculptures vivantes se mêlant à la peinture et à la viande selon une esthétique dionysiaque. Les corps sont à la fois toiles et pinceaux, accumulations de couleurs, de textures, de formes. Le film permet de se concentrer sur l'action, en la figeant tout en révélant sa propre mobilité, de fournir une documentation essentielle de cette énergie visuelle aux qualités expressives et expressionnistes révélées par la bande sonore mêlant des bruits urbains parisiens à des chansons Pop de l'époque. " L'explosion » matérielle et corporelle qui a lieu sur scène, transpire à travers le film qui permet de montrer de quelle manière l'action nécessite une sortie du cadre et se déroule également en dehors des limites scéniques. En témoignent les nombreux hors-champs qui rythment le film et montrent le dépassement des limites spatiales scéniques et en conséquence

\footnotetext{
${ }^{13}$ Carolee Schneemann raconte à plusieurs reprises de quelle façon un des membres du public a tenté de l'étrangler au cours de la représentation.

${ }^{14}$ Notes de l'artiste conservées dans ses archives au Getty Research Institute.

15 «I'm a painter extending the visual principles of painting into lived/activated time and dimensional space. »

${ }^{16}$ Carolee Schneemann réalise sa première pièce de " théâtre cinétique » en mai 1962 intitulée Glass Environment for Sound and Motion, au Living Theater de New York, dans laquelle elle ne performe pas encore.

17 Pierre Dominique Gaisseau, film 16mm, 1964.
} 
également filmiques. Edité par l'artiste, le film fut réalisé par Pierre Dominique Gaisseau, Schneemann performant sur scène. Ce n'est que pour le film Fuses qu'elle manipulera la caméra et la pellicule filmiques, à la fois à travers le montage et l'intervention picturale sur la bande de celluloïd. Le film, qui ne dure que quelques minutes, réalisé de la performance Meat Joy permet de se concentrer sur les aspects essentiels de l'œuvre. Les premières vues effectuées en plongée dévoilent le dessin formel de la performance créé par les corps en formation circulaire, motif dont le spectateur de l'action live n'est pas conscient de par son point de vue frontal. Le film apporte donc une vision particulière et originale de l'action. Puis la caméra adopte différents angles de captation, tant à la hauteur du champ visuel du spectateur que du point de vue des performeurs, la caméra se mêlant à eux, effectuant des vues d'ensemble du groupe mais également des plans plus rapprochés de chaque individu, transmettant ainsi l'appréhension des participants, performeurs et spectateurs, qui étaient censés se mélanger à Meat Joy. Les différents plans tournés se concentrent principalement sur les moments essentiels de l'action, à savoir " les rituels » effectués avec la viande, la peinture et les papiers. A ce moment, la caméra se mêlant au groupe, fait partie intégrante de la performance et est capable de participer au mouvement engendré par les corps et les matières.

Dans un entretien avec Nick Kaye, l'artiste rapporte que le fait d'être peintre et de pratiquer des formes artistiques autres, était considéré comme une "dévalorisation d'une forme par une autre ", ce qui n'aidait pas à la reconnaissance et à la légitimité recherchée par l'artiste. Pourtant la majorité des artistes masculins de l'époque développant la performance et surtout le happening qui venaient de domaines tels que la peinture ou la sculpture, s'adonnaient à une extension de la tactilité et de la matérialité de leur médium premier dans une dimension spatiale.

L'engagement avec la chair humaine et animale est influencé en partie par les théories du poète Michael McClure qui déclarait que tous les êtres vivants étaient de simples sacs de viande. Schneemann surmonte la charge implicite de narcissisme et d'exhibitionnisme afin d'expliciter la différence résidant entre le fait de montrer son corps et d'être son corps. Dans son échange épistolaire avec Clayton Eshleman, elle écrit: «I do not show my naked body! I am being my body » (Osterweil 136). Le corps et la nudité s'imposent comme une résistance politique et une transformation sociale. L'œuvre explore l'incarnation, la corporéité de la danse, la sensibilité manuelle de la peinture, dans des médiums de la durée comme le film et la performance. C'est une forme de prise de conscience, de vulnérabilité qui s'opère. La chair devient une source physiologique du contact avec l'autre, une esthétique de la participation, une révélation empathique comme le suggère Kaja Silverman (Flesh). Le corps s'affirme comme un système d'identification à l'autre et un lien de cohésion, de rassemblement, universel et humaniste. L'œuvre, et l'utilisation que l'artiste fait des corps, tente de surmonter les inégalités sociales et de faire prendre conscience des différences, des individualités identitaires et de l'altérité. Le film permet notamment de créer un cadre social d'identification. Face aux images des corps desinhibés, mêlant masculin et féminin dans une quasi-nudité, les différences affirment l'altérité et facilitent l'acceptation et la compréhension d'autrui.

La poésie corporelle tente de s'inscrire dans une forme de révolte. Cette gestualité rituelle et la célébration de la chair exubérante et sensible « engagent 
les sens, s'élargissant vers des relations inconnues et imprévues » (Elder 192). ${ }^{18}$ L'imagerie issue des rêves de l'artiste, de l'expérience charnelle et de l'énergie générée par le groupe lors des répétitions, se concentre sur l'essentiel du corps. ${ }^{19}$ La nudité artistique est utilisée contre la répression sociale, les régimes politiques autoritaires et adopte une forme de liberté et de libération de l'expression. ${ }^{20}$ Cette réflexion sur le pouvoir de la nudité s'étend à sa relation au public, initiée par Artaud. Marquée par son «théâtre de la cruauté ", Schneemann emploie cette esthétique alternative du moi et de son authenticité, amenant à une transcendance spirituelle. L'artiste et son corps, comme objet de souffrance, s'incarnent dans un théâtre corporel, expressif, faisant tomber le quatrième mur. Les spectateurs sont ainsi transformés en participants. La physicalité devient le primat de la pensée et permet de surpasser la séparation entre l'acteur et son rôle. La représentation et l'illusion disparaissent au profit de la présentation et de l'action. Amelia Jones rappelle que le corps de l'artiste devient véritablement visible à partir des années 1960 et donc potentiellement objet et sujet filmique à partir de cette même période. Dans cette forme d'autoreprésentation, la violence et l'affirmation du soi sont les thèmes les plus prégnants. Au-delà de l'enregistrement du corps, c'est l'enregistrement d'une image du corps, d'une présentation du " moi » mis en scène spécifiquement pour la caméra. L'image filmique produite est donc une représentation d'une représentation. C'est notamment ce que révèle le film tiré de la performance dont la représentation de la dimension collective de la sexualité permet de dénoncer le puritanisme de l'ordre établi et se révèle comme un moyen d'exorciser la violence faite aux corps: «Dans le travail de théâtre cinétique Meat Joy le corps voyant comme partie de l'événement de peinture, et dans Snows - les événements de photographie et film - deviennent centraux » (Ragona 36). ${ }^{21}$

Le théâtre cinétique est une façon de traiter les images de façon différente, de manière mouvante même si le support reste fixe, et de rendre compte d'une époque et de ses événements. L'artiste met en application ses développements au service du médium filmique et notamment dans l'œuvre Fuses. En ce qui concerne la représentation du corps, le film à l'inverse de la photographie n'impose pas un arrêt sur image mais un effet de continuité, de progression et de processus de l'œuvre et de sa création, permettant le détail et la décomposition du mouvement. Dans le cas de Schneemann, cette capacité de l'image en mouvement vient appuyer le lien constant qui existe entre l'œil, machine de vision, la caméra et le corps de l'artiste: «Mon exploration d'une image en mouvement veut dire simplement que sa réalisation coïncide avec son évocation » (Schneemann More than Meat 11). ${ }^{22}$ Le film permet la mise en image de l'événement mais explore également la possibilité qu'une partie de l'événement se réalise grâce à la présence de la caméra.

\footnotetext{
18 « engage our senses, expanding them into unknown and unpredictable relationships. »

${ }^{19}$ Ce rapport au corps et à la nudité en public trouve son origine dans le principe allemand de Freikörperkultur, prônant la connexion avec la nature et permettant de dépasser le tabou social. 20 Cette influence vient des théories de Norman O. Brown à travers sa relecture de la psychanalyse de Freud et la critique de la politique dans son ouvrage Love's Body de 1966.

21 «In Schneemann's kinetic theater works, Meat Joy (1964) and Snows (1967) the 'seeing body' as part of the event of painting, and in Snows - also the events of photography and film become central. »

22 My exploration of an image-in-movement means only that its realization supercedes my vocation of it. »
} 


\section{Matérialité du corps et tactilité du film}

Tourné avec son compagnon, le musicien et compositeur, James Tenney, Fuses $^{23}$ n'est pas le premier film dans lequel Schneemann est mise en scène. Le couple est filmé par Stan Brakhage dans Loving (1957) et Cat's Cradle (1959). Souhaitant reprendre possession de son corps, de son image, de sa féminité et de la représentation de sa sexualité, Schneemann s'empare de la caméra dans une utopie de la symbiose sexuelle masculin/féminin, d'une fusion quasiment androgyne voire hermaphrodite. Dans ses notes l'artiste esquisse un texte entre synopsis, scénario et déclaration d'intention:

Fuses a été réalisé (filmé et édité) sur une période de trois ans, inspiré par une relation de dix ans, utilisant l'amour sexuel de cette relation comme une image, comme une base, comme une peintre utilisant un paysage comme environnement. Je pense au film comme un paysage de chair, des changements de corps, un lit, une fenêtre, intérieur, extérieur, des cadres de portes à travers le cadre de l'œil de la caméra. Interrompre le film comme un œil de perception - la domination du rectangle. Utiliser les corps, nos corps, dans l'amour simplement, directement, sans conscience de soi. Et il n'y avait pas de cameraman ${ }^{24}$

Les images mises en scène, filmées et montées par l'artiste elle-même adoptent une esthétique kaléidoscopique représentant l'abandon sensuel devant une caméra, agent filmant et participant, révélant la peau de la pellicule. Le film devient une traduction picturale d'une sensation corporelle tout autant qu'un autoportrait artistique et féminin laissant apparaître l'intimité de l'artiste. ${ }^{25} \mathrm{~A}$ travers Fuses, Schneemann tente de remettre en cause « les limites du corps et de la nudité, afin de dépasser la paralysie psychologique et spirituelle » (Osterweil 147). ${ }^{26}$ Elle déploie une stratégie artistique admettant le corps comme zone potentielle d'identification:

Alors que Fuses crée un pont entre des modes de monstration écranique anciens et plus récents, écrit Amelia Jones, le film se trouve clairement à la fin d'une trajectoire moderniste de l'histoire de l'art et du film, qui a pris forme originairement dans les avant-gardes historiques des années 1910 et 1920 en Europe et refait surface après la Seconde Guerre mondiale, particulièrement dans le contexte du cinéma nord-américain

\footnotetext{
${ }^{23}$ Fuses est un film $16 \mathrm{~mm}$ Bolex, couleur, silencieux, de 22 minutes. En dehors de projections dans son studio, l'artiste montre son film pour la première fois en 1968 à Londres, lors de la conférence « Dialectics of Liberation ».

24 « Fuses was made (shot and edited) over a three year period, inspired by relationship of ten years, using the sexual love of that relationship as image as basic as a painter using landscape as basic environment. I think of the film as a flesh landscape shifts of bodies bed windows in and out doors rectangle of camera eye frame as paper canvas and to breaking in the film itself - as eye in perception - the domination of the rectangle. Using the bodies our bodies in loving simply, directly, without self-consciousness. And there was no camera man ». Notes de l'artiste conservées dans ses archives du Getty Research Institute.

${ }^{25}$ Lors de la réception de son film à la fin des années 1960 et au début de la décennie 1970, l'artiste se confronte à l'incompréhension de ses revendications par le mouvement féministe «anti-pornographie» mené notamment par Robin Morgan, célèbre pour son slogan « Pornography is the theory, rape is the practice ». L'incompréhension se porte particulièrement sur l'utilisation par Schneemann du corps féminin nu, et sur le désir qu'il peut susciter, dicté par une société patriarcale et phallocratique, dans laquelle la femme est objet de soumission. 26 « a way of shattering the limitations of the corporeal and political body, in order to move beyond the psychological and spiritual paralysis of the subject within hegemonic culture. »
} 
avec les travaux de réalisateurs comme Maya Deren et Stan Brakhage, avec lesquels Schneemann a travaillé avant de réaliser Fuses (Jones $134)^{27}$

L'artiste est notamment inspirée par l'aspect onirique et sensuel de Meshes of the Afternoon (1943) de Deren, dans lequel elle se met en scène avec la collaboration de son mari Alexander Hammid, « une méditation sur les rêveries d'une jeune femme caractérisée par un érotisme langoureux assombri par les suggestions d'un destin tragique imminent»(Elwes 92). ${ }^{28}$ En 1946, Maya Deren publie son ouvrage An Anagram of Ideas on Art, Form and Film. Elle y développe la théorie de « l'investigation verticale » qui permet de concevoir le film selon un axe vertical et non linéaire et horizontal. Cette conception du temps filmique apporte dynamisme et intensité, un temps non-chronologique qui peut parfois apparaître dissonant et déformé. Ces effets sont présents chez Deren puis chez Schneemann à travers le montage permettant une fragmentation de la perception. Il est donc évident que « les films de Maya Deren ont modelé en revanche le devenir du cinéma expérimental américain. Elle fut l'une des premières, sinon la première, à instaurer divers protocoles qui influeront sur les stratégies du cinéma expérimental: l'articulation en être la théorie et la pratique $\gg$ (Bullot 70-71).

Réalisé entre 1964 et 1967, Fuses est construit à partir de techniques développées notamment par Brakhage. Ce dernier s'intéresse au mouvement et au rythme apportés par un montage de scènes courtes. Tout comme Brakhage, Schneemann souhaite exploiter la dimension poétique et picturale du médium filmique ainsi que ses caractéristiques intrinsèques. Le travail d'un rendu flou, la caméra portative, la surexposition et la sous-exposition, l'utilisation de filtres, les multiples superpositions, une manipulation directe de la pellicule sont parmi les méthodes de Brakhage réutilisées par Schneemann. Mais l'artiste affirme que c'était essentiellement une réponse à l'annexion de la sexualité féminine par le réalisateur dans son film Window Water Baby Moving (1959), tourné lors de l'accouchement de sa femme. Dans son film, Schneemann décide de donner corporalité aux images en mouvement et à l'inverse de dévoiler toute la tactilité du corps filmé à travers la matérialité de la pellicule. Les limites entre privé et public sont troublées, la réalité rejoint la mise en scène de la performance artistique, et l'identité se révèle par la fusion des corps et des techniques. La représentation laisse place à la présentation de l'individu, de l'acte. Pourtant, l'effet de réflexion introduit par la caméra et son potentiel d'enregistrement vient inexorablement modifier le comportement individuel. Dans un entretien conduit par Kate Hug, l'artiste explique que la problématique majeure soulevée par Fuses est la représentation artistique de l'acte sexuel qui a, pendant de nombreuses années, été mise de côté à la fois par l'histoire du film, l'histoire de l'art et les mouvements féministes. Ce vide que vient combler Fuses est principalement dû à l'esthétisation, par le biais d'une pratique filmique atypique, d'images qui se voient sublimées par l'artiste. Le film adopte

\footnotetext{
27 « While Fuses straddles older and newer modes of screen culture, it still clearly occupies the tail-end of a modernist trajectory in the history of art and film, one originally taking shape in the historical avant-gardes of the 1910s and 1920s in Europe and resurfacing in the post-Second World War period, particularly in the context of North American cinema with the work of filmmakers such as Maya Deren and Stan Brakhage, with whom Schneemann worked for a period before making Fuses. »

28 « a meditation on a young woman's reveries characterized by a languid eroticism but overshadowed by intimations of impending doom. »
} 
donc une structure extrêmement formelle, tant dans son montage que dans sa production, permettant plusieurs niveaux de lecture mais positionnant le spectateur dans une situation de quasi-voyeurisme face au sujet privé et intime que dévoile la caméra. Une certaine ambiguïté émerge de cette situation : la composition formaliste et le rythme interne de l'œuvre sont contrebalancés par l'exacerbation de la tactilité du film à travers les images et les sensations corporelles révélées. Mais ce n'est que pour mieux surmonter les représentations de la nudité du corps féminin qui s'inscrivent, en-dehors du champ artistique, principalement dans des domaines soit médicaux soit pornographiques.

Montré pour la première fois dans le studio de l'artiste en 1965 à un public d'amis artistes, Fuses dépasse alors les attentes de la réalisatrice comme elle l'avoue à Laura Mulvey lors d'une conversation, en marquant une rupture en tant que vision artistico-érotique. En revanche, les séances suivantes vont s'avérer beaucoup plus controversées, voire incomprises et considérées comme outrageuses. En effet, en 1968 que ce soit au Festival de Cannes ou au Roundhouse de Londres, le film rencontre de nombreux problèmes de diffusion et de projection liés aux questions de censure. Envisagé par les uns comme n'étant pas suffisamment pornographique, au vu de leurs attentes, par les autres comme outrancier, Fuses témoigne d'une volonté de s'inscrire dans une historicité de libération et de féminisme du corps. L'œuvre va donc rencontrer une réception extrêmement contrastée, si ce n'est contrariée, par les groupes féministes qui refusent l'objectivation du corps féminin érotisé et sexualisé pour le regard masculin, allant jusqu'à reprocher un narcissisme prononcé dans cette auto-célébration de la chair. Pourtant, l'artiste s'inscrit dans une histoire artistique de la représentation du nu féminin et parvient à en dépasser les limites en faisant de son corps à la fois l'objet de la représentation, le sujet de l'œuvre, tout en étant elle-même l'artiste créant cette image.

L'acte sexuel du couple est filmé à plusieurs reprises, dans leur environnement intime, à travers le regard d'un témoin extérieur, celui de leur chat. Sont ajoutés des images de paysage et des symboles pouvant être connotés sexuellement. Une spontanéité se dégage des vues et plans, engendrée par des pertes de mise au point, des images floues, décadrées. Le film apparaît comme extrêmement pictural dans le choix des couleurs, dans sa forme et les dialectiques qui en découlent: entre chaleur et froid chromatique, entre intériorité et extérieur corporels, entre l'intimité privée et l'espace public. Dans son analyse du film, David James décrit: « La densité de sa construction, les images fragmentées des corps nus et le traitement égalitaire des démonstrations d'affection, Fuses se différencie de manière significative des autres représentations d'actes sexuels et plus particulièrement, la pornographie $\gg{ }^{29}$ En effet, à travers l'image filmique, Schneemann parvient à faire de l'acte sexuel un acte artistique, l'esthétisation du film se transmet à l'action de la représentation et l'identité des deux corps fusionne à l'écran.

Ne considérant pas son film comme un documentaire, l'artiste exprime sa déception face aux réactions d'incompréhension dans un entretien donné à Aviva Rahmani au sujet de la censure:

\footnotetext{
29 «The density of its construction, the fragmentary images of the naked body, and the egalitarian treatment of the lovemaking mark Fuses as significantly different from other representations of sexual acts, most notably, pornography. »
} 
Mon travail semble occuper une zone correspondant à un angle mort de l'art. Les réactions négatives dues à la sexualité de mon travail m'ont rendue furieuse. J'ai toujours considéré que j'avançais de manière progressive et cohérente. Dans Fuses, la nécessité était d'investir l'absence culturelle de la visibilité de l'intimité hétérosexuelle qui correspondait à ma propre expérience (Schneemann Imagining 215)

De plus, la réalisation de Fuses s'insère dans un contexte historique et politique nord-américain extrêmement vif. Ce qui explique que la représentation de la nudité et de l'acte sexuel ainsi que leur réception à travers le film se transforment en geste politique, revendiquant la liberté et dénonçant la violence et les abus dirigés vers les femmes et les autres minorités, au sein d'une société patriarcale.

Cette violence contemporaine est transcendée par les images filmiques des corps, la caméra entrant dans l'intimité des protagonistes, fragmentés, décadrés, perturbés par la matérialité artistique. L'artiste est engagée politiquement et fait notamment partie des groupes de revendication et de soutien anti-guerre du Vietnam. Amelia Jones revendique la position de Schneemann comme une artiste féministe majeure (Jones 126). Elle écrit que « Fuses n'est pas une simple exploration personnelle de l'érotisme, mais est lié aux vicissitudes de l'histoire politique. Du début au milieu des années 1960, Schneemann était à la fois une artiste et une activiste travaillant contre les actions du gouvernement américain au Vietnam » (Jones 129). ${ }^{31}$ L'auteure continue: « Alors que Fuses n'est pas un commentaire direct sur le Vietnam, son approche viscérale de l'incarnation est à mettre en relation avec la tentative stratégique dans ses autres projets de dévoiler la violence politique et militaire perpétrée par les troupes américaines à l'étranger » $(130) .{ }^{32}$

Ainsi, parallèlement au travail sur Fuses et en réaction à la guerre du Vietnam, l'artiste réalise le film Viet-Flakes ${ }^{33}$ en 1965 à partir d'images provenant de journaux européens ayant enregistré le conflit armé. Le film est projeté lors de la performance Snows. A cette époque, la projection filmique sur scène lors de pièces chorégraphiées se fait de plus en plus courante. Quant au film de Schneemann, il est « réalisé avec l'utilisation d'une loupe entre la caméra et les images de magazine des victimes vietnamiennes, présent tout au long de Snows, ajoutant un effet surréel, aux flashs et cadres fixes et lumières stroboscopiques » (Ragona 37) ${ }^{34}$ L'artiste révèle la valeur de la chair comme matériau artistique

\footnotetext{
${ }^{30}$ « My work seems to occupy a zone corresponding to the art world's blind spot. The sexually negative reactions to so much of my work has enraged me. I always felt I was doing the obvious next step. In Fuses, the necessity was to investigate the absence in my culture of visual heterosexual intimacy that corresponded to my own experience. »

${ }^{31}$ « But Fuses is hardly simply a personal exploration in eroticism unattached to the vicissitudes of political history. By the early to mid-1960s Schneemann was an artist and simultaneously an activist working against the US government's actions in Vietnam. »

32 "While Fuses is in no way directly a commentary on Vietnam, its visceral approach to embodiment clearly parallels Schneemann's strategic attempt in her other projects to unveil the political and military violence perpetrated by US troops abroad. »

${ }^{33}$ Viet-Flakes est un film 16 mm réalisé entre 1965 et 1967, en noir et blanc, teinté bleu, sonorisé par James Tenney. Le titre fait référence au Vietnam et au symbole commercial américain des Corn Flakes.

34 « shot by using a magnifying glass that mediated between her camera and magazine images of Vietnamese victims, figured prominently throughout Snows, adding a surreal, haunting stutter to the live flash-edits or freeze frames simulated by ongoing strobe lights. »
} 
et les caractéristiques que le corps peut apporter à l'œuvre. Snows affirme cette valeur charnelle politique de corps marqués par la violence de la guerre. ${ }^{35} \mathrm{Le}$ film se superpose aux corps mouvants et performants, générant une fusion entre les corps scéniques et filmiques. Dans la collision des corps et des images, le film apparaît comme un élément structurel, mettant en avant le corps du performeur et sa résistance, seule réponse à la guerre et aux atrocités subies par les autres corps. Mathilde Roman explique les problèmes que peut susciter la projection scénique: «En acceptant les contraintes du spectacle vivant, la frontalité du rapport du spectateur à l'œuvre et une durée imposée, ils affirment la complicité première de l'image vidéo avec l'espace scénique. Projetée sur scène, elle n'a pas un statut différent, et c'est ce qui explique la difficulté pour les artistes à opérer un véritable déplacement de leur œuvre en l'intégrant à un spectacle » (Roman 154). C'est par le biais de cette projection que la violence physique et psychique est retransmise au spectateur. Cette violence intervient à différents niveaux, à la fois dans les corps mutilés par la guerre, dans les corps scéniques malmenés par la performance, le corps pelliculaire du film lors de sa création et le corps spectatoriel dans la réception d'une œuvre mise en scène et en espace pour le bouleverser. En effet, les corps photographiés que Schneemann filme disent toute l'atrocité de la guerre. Mais elle parvient à créer une tension à travers le décalage entre image fixe, qui apporte une valeur indicielle, et le mouvement de la caméra qui filme les photographies. Les corps sont à la fois figés et mobiles, et leur mutilation, souffrance et déformation apparaissent et disparaissent au gré des effets bougés, de flickering, ${ }^{36}$ de flash, de flou qui ne laissent les images visibles que pour un instant furtif, à tel point qu'il devient difficile de savoir ce que l'on voit et ce que l'on croit voir. La méthode est similaire à celle de Fuses: un montage à la fréquence saccadée, une multitude de motifs qui s'entre-aperçoivent quelques secondes et se répètent pour autoriser la vision de l'inconfortable ou de l'insoutenable, mais demeurent indiscernables. Basé sur des images documentaires, Viet-Flakes est tout sauf un film-document. Il déplace ainsi ses aspects et caractéristiques vers la performance et les corps scéniques lors de sa projection à la fin de Snows. Concernant Fuses, David Levi Strauss confirme d'ailleurs:

L'action est non-séquentielle, non-narrative, et ne se dirige pas vers un point culminant. La manipulation physique de Schneemann de la pellicule filmique - brûlée, cuite, coupée, grattée, peinte, colorée, trempée dans l'acide, exposée au climat - est utilisée pour véhiculer les images à travers le corps. Cette intégration ou fusion du sujet et de la méthode, du fait et de la facture, est au centre de la pratique de Schneemann (Levi Strauss $30)^{37}$

\footnotetext{
${ }^{35}$ La performance est présentée dans le cadre de la « Week of Angry Arts », à New York. Parmi les photographies utilisées par Schneemann se trouvent celles montrant les victimes du napalm publiées dans l'article de William F Pepper, «The Children of Vietnam », dans le magazine Ramparts en janvier 1967.

${ }^{36}$ Effet de clignotement, utilisé également par Brakhage ou Paul Sharits.

37 « The action is non-sequential, non-narrative, and doesn't build a climax. Schneemann's physical manipulation of the film stock - burning, baking, cutting, scratching, painting, coloring, dripping it in acid, leaving it outside in the weather - serves to bring the images through the body. This integration or fusing of subject and method, fact and facture, is at the center of Schneemann's practice. »
} 
Ces techniques et méthodes filmiques vont donc concourir à mettre en place une œuvre repoussant les limites visuelles du spectateur et brisant « la causalité narrative » (Bullot 55).

A travers la subversion de la narration traditionnelle et la subjectivité des séquences, Fuses répond donc aux caractéristiques développées par Laura Marks en terme de tactilité et d'haptique: «La perception haptique est définie comme la combinaison des fonctions tactiles, cinétiques et proprioceptives. La façon dont on expérimente le toucher à la surface et à l'intérieur de nos corps. Alors qu'avec la visualisé haptique, les yeux fonctionnent comme des organes du toucher » (Marks 2). ${ }^{38}$ Cette dimension accroit la relation corporelle du spectateur avec l'image et donc son identification à l'œuvre. D'après Vivian Sobchack, le visionnage d'un film étant un échange de deux corps: celui du spectateur ainsi que celui du film. La « relation intersubjective » qui en découle confirme l'aspect « haptique érotique » de Fuses: « Ce qui est érotique à propos de la vision haptique, écrit Laura Marks, peut être décrit comme le respect d'autrui, et la perte de soi concomitante en présence de l'autre » (Marks 20). ${ }^{39}$ La fusion de la matière du corps avec celle du film renforce également l'identification de l'artiste avec son œuvre faisant de Fuses « un poème charnel autobiographique et érotique contemporain. » Jones ajoute que «l'engagement haptique avec le corps filmique permet au spectateur de s'ouvrir à une relation viscérale avec les corps de Schneemann et de son amant » (Jones 146). ${ }^{40}$

C'est pourquoi Jean-Michel Bouhours peut désormais affirmer que «Carolee Schneemann, est une pionnière dans l'art de la performance multimédia ou intermédia - son film Fuses (1965) explorant les limites du voyeurisme cinématographique est un classique » (Bouhours 338). En effet, Schneemann parvient à s'inscrire au cœur de la transversalité artistique en se positionnant dans le prolongement de l'histoire de l'art à travers l'utilisation de motifs comme la fenêtre, indice de la perspective renaissante, le bouleversement des hiérarchies picturales modernes, en incluant à la fois le nu féminin et masculin, des paysages, une forme d'autoportrait, une scène de genre de sa vie quotidienne ainsi que des références sous-jacentes et symboliques du contexte historique. Mais elle déborde également cette histoire de l'art en innovant du point de vue des techniques filmiques: gros plans détruisant le motif, montage clignotant, colorisation et inversion du sens de l'image, utilisation d'une caméra suspendue. L'œuvre de Schneemann parvient à dresser un parallèle entre la chair violentée dans la réalité et la chair exposée à la vue de tous, au cœur d'un acte relevant de l'intimité ou d'une action artistique.

\section{Genre artistique et identité féminine}

Peggy Phelan lie cette réflexion artistique au thème de l'identité:

\footnotetext{
38 « Haptic perception is usually defined as the combination of tactile, kinesthetic, and proprioceptive functions, the way we experience touch both on the surface of and inside our bodies. In haptic visuality, the eyes themselves function like organs of touch. »

39 «What is erotic about haptic visuality, then, may be described as respect for otherness, and concomitant loss of self in the presence of other. »

40 « the haptic engagement with the body of the film, in turn, opens the viewer to a visceral relationship with the bodies of Schneemann and her lover. »
} 
qui ne peut pas résider dans le nominatif ou dans la vision du corps. L'identité émerge de l'échec du corps à exprimer sa complétude et l'impossibilité à transmettre une signification exacte. L'identité est perceptible uniquement à travers la relation à l'autre ... Dans une déclaration d'identité et d'identification, il y a toujours une perte, la perte de ne pas être l'autre, et pourtant de dépendre de cet autre dans le fait d'être et de se reconnaître (Phelan 132) ${ }^{41}$

Ce type d'identification révèle de ce que Marks définit comme skin of the film ou visualité haptique du film : «En manipulant la chair du celluloïd, brûlé, cuit, coupé et peint, plongé dans l'acide, et construit selon des couches de collage..., Schneemann rompt avec les conventions du texte filmique » (Jones 135). ${ }^{42}$ Ainsi, en ajoutant de la texture à son matériau filmique et en pratiquant un montage non linéaire, l'artiste apporte un supplément de tactilité visuelle à son œuvre. Les images en mouvement et l'érotisme qui s'en dégage s'inscrivent dans une lecture bipartite. Chair filmique et chair corporelle fusionnent dans une tentative de redéfinir les codes de représentation de l'identité artistique et sexuelle.

En effet, que les revendications de l'artiste soient d'ordre politique, artistique ou autobiographique, l'iconographie de la nudité du corps féminin est à la source de l'expression de son propos. Ainsi les trois œuvres Eye Body (performance photographique de 1963), Meat Joy (performance collective de 1964) et Fuses (film érotico-autobiographique de 1964) forment un trio éroticoartistique dans lequel le corps nu de l'artiste est le support de ses revendications: « Schneemann travaille à perturber les limites esthétiques et culturelles entre la peinture et les autres matériaux et questionne les conventions acceptées du genre » (Serra Ramey 104). ${ }^{43}$ Dans sa capacité à représenter le mouvement et la physicalité, le corps se fait matériau et instrument de vision dont la prise de contrôle à travers la création artistique expose les interdits collectifs et culturels. En 1977, Schneemann rejoue la performance Interior Scroll ${ }^{44}$ lors de son intervention au Telluride Film Festival dans le cadre du programme "The Erotic Woman » organisé par Brakhage. L'artiste déroule alors de son vagin un long morceau de papier sur lequel est inscrit le texte «I Met a Happy Man » provenant de la bande sonore de son œuvre Kitch's Last Meal. Ce discours énonce notamment une critique masquée, dirigée contre Annette Michelson critique de film, décrite ici par Schneemann sous les traits d'un réalisateur structuraliste $^{45}$ et qu'elle qualifie de «dragon » dans une correspondance avec Scott MacDonald (Stiles 343). En effet, Schneemann reproche à Annette

\footnotetext{
${ }^{41}$ « Identity cannot, then, reside in the name you can say or the body you can see... Identity emerges in the failure of the body to express being fully and the failure of the signifier to convey meaning exactly. Identity is perceptible only through a relation to another...In that declaration of identity and identification, there is always loss, the loss of not-being the other and yet remaining dependent on that other for self-seeing, self-being. »

42 « By manipulating the flesh of the celluloid itself 'burning, baking, cutting, and painting it, dipping my footage in acid, and building dense layers of collage ... held together with paper clips', Schneemann broke apart the conventional film text, enacting her own agency as a making subject. »

${ }^{43}$ « Schneemann works to disrupt aesthetic and cultural limitations between painting and its extended materials and to question acceptable gendered conventions. »

${ }^{44}$ Interior Scroll est interprétée en 1975 lors du festival «Women Here and Now » à East Hampton, puis en 1977 au « Telluride Film Festival » dans le Colorado.

${ }^{45}$ Le réalisateur de film structuraliste a d'ailleurs souvent été identifié, à tort, comme étant Anthony McCall.
} 
Michelson d'avoir toujours évité de regarder ses films. Dans une lettre à Daryl Chin, Schneemann écrit: "Annette n'a absolument jamais vu aucun de mes films et n'a donc bien entendu jamais écrit à leur sujet et lorsque que nous nous sommes croisées à une conférence, je lui ai dit que ce serait enfin l'occasion de voir Fuses, mais ... Annette a préféré rentrer dormir à l'hôtel » (Stiles Correspondance 222). ${ }^{46}$

Dans ce contexte de contestation et de revendication à la fois politique et artistique, l'artiste exprime sa volonté de dénoncer les conditions de création et de réception des films réalisés par des artistes femmes et l'atmosphère misogyne à laquelle elles doivent faire face. Dans son texte introductif rédigé à l'occasion du festival, Schneemann s'interroge:

Nous devons nous demander comment les hommes sont parvenus à ce que leur création, invention, interprétation et observation de la femme soient les seules authentiques. Pourquoi lorsque les femmes définissaient, exploraient et structuraient leur propre monde créatif, cela était dénué de substance, de sérieux - que seuls les hommes parmi les hommes pouvaient établir la culture dominante ? Pourquoi Brontë, Eliot et Sand écrivaient sous des pseudonymes masculins ? Pourquoi Woolf était dévalorisée, traitée comme une auteure de seconde zone ? Pourquoi les innovations de Dorothy Richardson dans le langage comme une forme de mémoire spatiale ont été ignorées ? Pourquoi les rares découvertes de la vision féminine qui ont été importantes à leur époque, ont été perdues dans l'histoire ? ${ }^{47}$

Levi Strauss s'interroge à ce propos: « Est-ce que la suppression, l'exclusion et l'inattention des artistes femmes aux imaginations radicales se sont transformées en notion d'histoire de l'art et par extension à l'histoire du film et de la critique ? » (Strauss 320). ${ }^{48}$ C'est ainsi que la performance s'impose sous son aspect politique et marginal permettant le dévoilement de l'identité à la fois artistique et féminine. Mieke Bal définit la performance comme étant: « initialement un mot, la performativité un concept théorique. La performance - l'exécution unique d'un travail - est d'un différent ordre que la performativité, un aspect d'un mot - ou d'un travail - qui fait ce qu'il dit. Ainsi, la performance n'est pas la performativité, ce qui importe est la matérialité, du concret à l'abstrait, ou du terme objectif au terme théorique » (Bal 168). ${ }^{49}$ Le film permet

\footnotetext{
46 « Annette has never never ever ever seen any any film of mine never never ever and so of course has never never never written about them and then guess what when we are at buffalo women in film conference I said this is good Annette now at least you get to see Fuses but... Annette slept in her motel room. »

47 «We have had to ask how did the men come to insist that their creation, invention, interpretation and observation of woman was the only authentic one ? Why when women defined, explored and structured their own creative worlds was this denied weight, seriousness - that only men among men could establish the mainstream of culture ? Why did Bronte, Eliot and Sand write under male pseudonyms? Why was Woolf treated as a trivializer, a secondary author. Why were Dorothy Richardson's innovations in language as a form of special memory ignored? Why were the rare breakthroughs of female vision which were granted importance in their own time, lost in subsequent history ? » Notes de l'artiste conservées dans ses archives au Getty Research Institute.

48 «Is the suppression, exclusion, and neglect of women artists with radical social imaginations somehow built into the notion of art history and by extension film history and criticism ? »

49 «Performance, for me, was initially just a word, performativity a theoretical concept. Performance - the unique execution of a work - is of a different order than performativity, an aspect of a word - or work - that does what it says. Hence, performance is not performativity
} 
de révéler ces deux aspects de l'œuvre, à la fois la performance et la performativité, l'aspect concret, matériel autant que l'aspect abstrait et théorique de l'œuvre. L'auteure continue: «Au lieu, sa théâtralité emphatique invite le spectateur à jouer un rôle - à performer. Le jeu devient l'acte où la performance et la performativité interagissent sans pour autant fusionner » (170). ${ }^{50}$ Mais c'est justement le film qui va permettre cette fusion, ou tout du moins cette co-existence et transformer le spectateur en performeur. En conséquence, Kaja Silverman soulève le lien entre performance et identité, en utilisant le terme "d'identification hétéropathique» (Silverman Male Subjectivity 264), qui permet à l'individu de se détacher de son propre corps pour se diriger vers l'autre et s'identifier. Le corps devient un langage à part entière et le corps filmé est lui-même un nouveau médium d'interprétation. Comme nouveau régime d'identification, le corps remet en question la stabilité de l'identité et du " moi » ainsi que de la notion d'individu. La performance permet le changement et la transformation du corps tout autant que sa représentation à travers l'image filmique. L'enregistrement de la caméra révèle le corps et sa valeur ontologique. Laura Mulvey souligne que c'est en priorité le regard masculin qui va changer la femme en objet et par extension son corps, lieu de questionnement de l'identité du genre et de la sexualité. Pourtant Emilie Bouvard rappelle que «Carolee Schneemann a de fait peu exposé avec les artistes féministes dans les années 1970. Elle est absente des Etats-Unis au moment où le mouvement féministe se structure: elle vit à Londres entre 1969 et $1973 »$ (Bouvard 58). Ce sont pourtant bien des revendications féministes que développe l'artiste, un égalitarisme du genre et artistique, une dénonciation de la société patriarcale à travers l'analyse de la sexualité lorsqu'elle écrit:

C'est dans notre intérêt à tous de recevoir pleinement la vision des femmes par des femmes. En fait, c'est l'artiste homme qui historiquement s'est castré en bannissant et gardant à distance l'entière participation de la sensibilité féminine et des formes culturelles. Les traductions patriarcales ont séparé le corps et l'esprit, la romance et la dégradation, le sacré et le profane ${ }^{51}$

Jay Murphy atteste que « le travail de Carolee Schneemann dans divers médias invariablement prend comme terrain d'exécution son propre corps, mettant en avant ses rêves, sa sensorialité, et ses explosions physiologiques d'inspiration ... Pour l'art féministe, dans un sens plus général, Schneemann reste une pionnière problématique » (Murphy 227). ${ }^{52}$ Car l'artiste a toujours utilisé son corps dans une démarche politique et proto-féministe. Elle participe au changement de la représentation, de l'utilisation et de l'appréhension du corps entre les années 1960 et aujourd'hui, ainsi qu'à l'évolution de la perception du corps par la culture populaire dans sa possibilité de repousser les limites. L'artiste est ainsi à la fois l'image, le sujet et la créatrice de l'image, recréant

what matter is the materiality, the concrete to the abstract, or the object term to the theoretical term. »

50 « Instead, its emphatic theatricality invites the viewer to 'play a part' - to perform. Playacting thus becomes the act where performance and performativity interact without merging. "

${ }^{51}$ Texte introductif pour le programme « Erotic Films By Women » du Telluride Film Festival, 4 septembre 1977.

52 « Carolee Schneemann's work in various media invariably foregrounds the processes of her own body, highlighting its dreams and its sensory and physiological bursts of inspiration ... for feminist art in a more general sense, Schneemann remains a problematic pioneer. » 
l'identité de l'œuvre et de l'artiste en les confondant au sein d'un travail en perpétuelle transformation.

Pour conclure, il semblerait donc que dans son implication au contexte de son époque, Schneemann, comme l'explique Bouvard, parvient à : « faire de l'art, à écrire l'Histoire et l'histoire de l'art de manière à déjouer une linéarité refoulante » (Bouvard 63). Même lorsque les images de l'actualité soulèvent le problème de la difficulté à regarder de telles images, comme dans l'œuvre Terminal Velocity (2001), créant comme Viet-Flakes une sorte de Sublime contemporain. L'artiste se positionne comme témoin d'un événement historique. Elle développe ainsi un « potentiel animistique », à partir d'images qui n'apparaissent qu'aux limites du perceptible, du regardable et du soutenable. Comme l'explique Annabelle Ténèze, conservatrice au Musée d'art contemporain de Rochechouart, l'artiste parvient à révéler « une vérité historique tangible dans une narration volontairement sans récit » (Ténèze 26). Pour cela, elle met en place "une expression physique décomplexée, l'exploration des sentiments les plus extrêmes, la vertu cathartique du geste 'hors limites', y compris la reproduction mimétique des pires actes » (Ténèze 29); et parvient à incarner l'Histoire à travers l'œuvre, même si pour cela elle doit « représenter ce qui est inimaginable, irreprésentable, injustifiable » (29). Schneemann dévoile « une chronique du présent écrite grâce à une acuité et une intuition continuelle d'une époque et de sa transformation » (35). Comme l'explique l'historienne de l'art Kristine Stiles, la pratique artistique de:

Schneemann a constamment élargi les discours contemporains vers un vocabulaire visuel et textuel des formes et des idées convergeant vers et expressives des thèmes centraux de son époque: l'autorité et la fragilité du corps humain à la fin du $\mathrm{XX}^{\mathrm{e}}$ siècle; la signification du genre en repensant et reconstituant les conditions sociales de l'épistémologie occidentale; les connexions ténues entre la maladie, la mort, la perte et l'amitié; l'amour et sa violence; ... la représentation et l'étude de la sexualité (Stiles At Last! 231) ${ }^{53}$

Tel l'outil filmique, Carolee Schneemann s'affirme comme étant «à la fois réceptrice et émettrice » (Delpeux 33). Elle reste inclassable, mais c'est justement cette marginalité qui la place entre l'histoire de l'art, les études cinématographiques et l'histoire culturelle, dans un environnement social complexe. Il est fondamental de noter qu'en 1970 paraît l'ouvrage de Gene Youngblood Expanded Cinema dans lequel il étudie la transformation du médium et son application dans d'autres domaines artistiques. Fuses y est mentionné comme incarnant « un nouvel érotisme polymorphe » (Youngblood $84) .{ }^{54}$

\footnotetext{
53 « Schneemann consistently extended contemporary discourses into a visual and textual vocabulary of forms and ideas convergent with and expressive of the central themes of her time: the authority and fragility of the human body in the late twentieth century; the significance of gender in rethinking and reconstituting the social conditions of Western epistemology; the tenuous connections between disease, death, loss, and friendship; love and its fury;...the representation and study of sexuality. »

54 « the new polymorphous eroticism.»
} 


\section{Bibliographie}

Aquin, Stéphane. Village Global: les années 60. Gent: Éditions Snœck / Musée des Beaux-Arts de Montréal, 2003.

Bal, Mieke. " Setting the stage: the subject mise-en-scène. » Art of Projection. Stan Douglas et Christopher Eamon (dir.). Stuttgart: Hatje Cantz, 2009. 167-181.

Blaetz, Robin. Women's Experimental Cinema. Durham: Duke University Press, 2007.

Bouhours, Jean-Michel. « Au-delà de l'écran... L'invisible et le horschamp ». Hors limites : l'art et la vie, 1952-1994. Jean de Loisy (dir.). Paris : Centre Georges Pompidou, 1994.336-343

Bouvard, Emilie. "Carolee Schneemann. Féminisme et Histoire». Then and Now. Carolee Schneemann. Arles : Analogues, 2013. 54-65.

Bullot, Erik. Sortir du cinéma. Histoire virtuelle des relations de l'art et du cinéma. Genève: Mamco, 2013.

Cameron, Dan. «In the Flesh. » Cat. expo. Carolee Schneemann, Up to and Including her Limits. New York: The New Museum of Contemporary Art, 1996. 7-14.

Delpeux, Sophie. Le Corps-caméra: Le performer et son image. Paris : Textuel, 2010.

Douglas, Stan et Christopher Eamon. Art of Projection. Ostfildern: Hatje Cantz, 2009.

Elder, R. Bruce. « Dream Flesh ». Carolee Schneemann. Unforgivable. Kenneth White (dir.). Londres: Black Dog Publishing, 2016. 192-207.

Elwes, Catherine. Installation and the Moving Image. London: Wallflower Press, 2015.

Frederickson Kristen. Singular Women. Writing the Artist. Berkeley: University of California Press, 2003.

Halsall, Francis. Rediscovering Aesthetics. Redwood City: Stanford University Press, 2009.

Harper, Glen. Interventions and Provocations: Conversations on Art, Culture, and Resistance. New York: State University of New York Press, 1998.

James, David. Allegories of Cinema: American Film in the Sixties. Princeton: Princeton University Press, 1989.

Jones, Amelia. Self/Image, Technology, Representation, and the Contemporary Subject. Londres: Routledge, 2006.

---. « Screen eroticisms: exploring female desire in the work of Carolee Schneemann and Pipilotti Rist ». Screen/Space: The Projected Image in Contemporary Art. Tamara Trodd et Sam Lackey (dir.). Manchester: Manchester University Press, 2011. 126-144.

Joseph, Branden W. «Sparring with the Spectacle. » Anthony McCall: The Solid Light Films and Related Works. Evanston: Northwestern University Press, 2005. 35-79.

Lavigne, Emma. Danser sa vie. Paris: Centre Georges Pompidou, 2011.

Levi Strauss, David. « Love Rides Aristotle Through the audience: Body, Image, and Idea in the Work of Carolee Schneemann. » Carolee Schneemann: Up to and Including her Limits. New York: The New Museum of Contemporary Art, 1996.

Marks, Laura. Touch: Sensuous Theory and Multisensory Media. Minneapolis: 
University of Minnesota Press, 2002.

Murphy, Jay. « Assimilating the unassimilable: Carolee Schneemann in relation to AntoninArtaud ». Imaging her erotics. Carolee Schneemann (dir.).

Cambridge: MIT Press, 2002. 224-31.

Osterweil, Ara. « On Fuses ». Carolee Schneemann. Precarious. Vienne: Schlebrügge, 2013.136-51.

Ragona, Melissa. «A Technological Interiority ». Carolee Schneemann. Precarious. Vienne: Schlebrügge, 2013. 256-79.

Roman, Mathilde. On Stage, la dimension scénique de l'image vidéo. Blou: Le Gac Press, 2012.

Schneemann, Carolee. Imaging her Erotics. Cambridge: MIT Press, 2002.

---. More Than Meat Joy: Complete Performance Works and Selected Writings. Kingston: McPherson and Co, 1979.

---. « Seasonal Fractional Political Idiosyncratic Aesthetics. » Rediscovering Aesthetics: Transdisciplinary Voices From Art History, Philosophy, and Art Practice. Francis Halsall, Julia Jansen et Tony O'Connor (dir.). Stanford: Stanford University Press, 2009. 213-224.

Serra, Mary Magdalene et Kathryn Ramey. « Eye/Body. The Cinematic Paintings of Carolee Schneemann. » Women's Experimental Cinema. Robin Blaetz (dir.). Durham: Duke University Press, 2007. 103-126.

Silverman, Kaja. Flesh of My Flesh. Stanford: Stanford University Press, 2009.

---. Male Subjectivity at the Margins. London: Routledge, 1992.

Sobchack, Vivian. The Address of the Eye: A Phenomenology of Film Experience. Princeton: Princeton University Press, 1991.

Stiles, Kristin. « At Last! A Great Woman Artist: Writing about Carolee Schneemann's Epistolary Practice. » Singular Women. K Frederickson et SE Webb (dir.). Berkeley: University of California Press, 2003. 213-237

--- Correspondance course: an Epistolary History of Carolee Schneemann and her Circle. London: Duke University Press, 2010.

Strauss, David Levi. « Love Rides Aristotle Through the audience: Body, Image, and Idea in the Work of Carolee Schneemann ». Carolee Schneemann, Up to and Including her limits. New York: New Museum of Contemporary Art, 1996. 26-34

Ténèze, Annabelle. " Entretien de Carolee Schneemann. Cat.expo. Then and Now. Carolee Schneemann. » Musée départemental d'art contemporain de Rochechouart, 4 octobre- 15 décembre 2013, Arles: Analogues, 2013.

Trodd, Tamara. Screen/Space. The Projected Image in Contemporary Art. Manchester: Manchester University Press, 2011.

Warr, Tracey. Le corps de l'artiste. Londres: Phaidon Press, 2005.

White, Kenneth. " Meat system in Köln ». Carolee Schneemann. Unforgivable. Kenneth White (dir.). London: Black Dog Publishing, 2016.

Youngblood, Gene. Expanded Cinema. Boston: EP. Dutton, 1970. 